\title{
Immunizations: three centuries of success and ongoing challenges
}

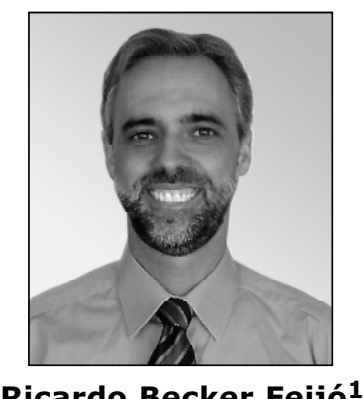

Ricardo Becker Feijó ${ }^{1}$
In the last few decades, immunization - one of the greatest breakthroughs in health sciences - has increasingly gained significant ground all over the world. Advances in general sciences, microbiology, pharmacology and immunology have, together with results of epidemiology and sociology studies, demonstrated the remarkable impact of vaccines on society and the importance of vaccination in health promotion and disease prevention.

In the beginning of the $17 \mathrm{~h}$ century, smallpox was one of the most devastating communicable diseases in the world; it affected most individuals before they reached adulthood, and had high mortality rates. Lady Mary Montagu, wife of the British ambassador in Istanbul at the time, observed that the disease could be avoided by using a technique adopted by Muslims, who inoculated dried pus from smallpox pustules obtained from an infected patient into the skin of healthy individuals. This procedure, known as "variolation," probably originated in China; later, it was

1. Professor adjunto de Pediatria, Faculdade de Medicina, Universidade Federal do Rio Grande do Sul (UFRGS), Porto Alegre, RS, Brasil. Doutor, UFRGS, Porto Alegre, RS, Brasil.

2. Professor assistente, Faculdade de Ciências Médicas, Santa Casa de São Paulo, São Paulo, SP, Brasil. Membro, Comissão Permanente de Assessoramento em Imunizações, Secretaria da Saúde do Estado de São Paulo, São Paulo, SP, Brasil. Coordenador, Serviço de Infectologia Pediátrica, Hospital São Luiz, São Paulo, SP, Brasil.

Suggested citation: Feijó RB, Sáfadi MA. Immunizations: three centuries of success and ongoing challenges. J Pediatr (Rio J). 2006;82(3 Suppl):S1-3.

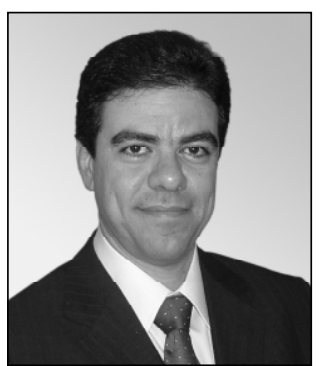

Marco Aurélio P. Sáfadi²

taken to Western Europe. Although it led to several cases of death due to smallpox, it was used in England and in the United States until the beginning of investigations by British physician Edward Jenner, whose research results were published in the study Variolae Vaccinae in 1798. Dr. Jenner studied peasants who developed a benign condition known as "vaccinia" due to their contact with cowpox, and his investigation resulted in the development of the first immunization techniques. Although lacking the bioethics principles later developed, his contribution to the history of medicine is undeniable. Nevertheless, the cause-effect relationship between the presence of pathogenic microorganisms and diseases was only established by Louis Pasteur and Robert Koch around 1870. To honor Jenner, Pasteur named "vaccine" (after the "vaccinia virus" in Jenner's investigations) any preparation of agents to be used for immunization against an infectious disease. In 1885, Pasteur developed the vaccine against human rabies, a landmark that established the beginning of a new era.

International organizations, such as the World Health Organization (WHO) and the Pan-American Health Organization (PAHO), underscore the global effect of immunizations and the importance of eradication of diseases at a world level, such as in the case of smallpox, which was last reported in 1977. By means of defined strategies and goals, the WHO and the PAHO provide direct support to countries to conduct their vaccination activities. 
In Brazil, the Programa Nacional de Imunizações (PNI - Brazilian National Immunization Program), created in 1971 , is a world reference. It has organized vaccination campaigns in other countries, such as East Timor, assisted immunization programs in Palestine, the West Bank and the Gaza Strip, and established technical cooperation programs in many countries. Its planned and systematic actions in Brazil led to the eradication of smallpox in 1973 and poliomyelitis in 1989, and to the control of measles, neonatal tetanus, severe forms of tuberculosis, diphtheria, tetanus and pertussis. Measures have been implemented to control mumps, rubella, congenital rubella syndrome, hepatitis $\mathrm{B}$, Haemophilus influenzae type $\mathrm{b}$ invasive infections and influenza, as well as pneumococcal infections and their complications in the elderly. A recent pioneering decision has been made to incorporate the oral vaccine against rotavirus to the PNI. The Special Immunobiology Referral Centers (CRIE - Centros de Referência para Imunobiológicos Especiais) in Brazil also supply immunobiologic products indicated for specific situations and population groups. Currently, the PNI adopts a policy of establishing partnerships and granting incentives to the technological upgrading of national production facilities, and most of the immunobiologic products used nationwide are produced in Brazil. ${ }^{3}$

The direct and indirect benefits generated by immunization programs are unequivocal and surprising: there is plenty of evidence showing their potential to reduce mortality among children and improve health and well-being in communities. They also generate savings for the whole society, both by reducing costs with medical visits, treatments and hospitalizations and by lowering school and job absenteeism. Therefore, the great challenge that we all face now is to support, by means of education, information and awareness raising, actions that expand the reach of immunizations to all communities. As a contribution to such goal, Jornal de Pediatria presents this Vaccination Supplement with up-to-date articles written by renowned national immunization researchers that share their experience on this topic and discuss controversies that affect our pediatricians' daily practices.

In the first article in this Supplement, the authors discuss the criteria adopted to prepare a "Vaccination schedule for childhood and adolescence," and compare different recommendations. In "Acellular pertussis vaccines for adolescents," the authors point to the possibility of controlling the increase in the number of cases of pertussis among adolescents and adults by using a single dose tetanus, diphtheria, acellular pertussis vaccine (Tdap) with a specific formulation for individuals older than 10 years to be administered in place of tetanus and diphtheria booster immunization in adolescence.

In "Vaccines against rotavirus and human papillomavirus (HPV)," the authors summarize the strategies used to obtain safe and effective vaccines against rotavirus and HPV. They report that the use of vaccines against rotavirus for immunization of 60 to $80 \%$ of the children in the next 10 years may reduce hospitalizations and deaths due to rotavirus in at least $50 \%$. They also stress that safe and effective vaccines against HPV would be important tools in the prevention of cervical cancer all over the world, particularly in developing countries such as Brazil. 4

In the article "Meningococcal conjugate vaccines: efficacy and new combinations," the authors discuss the recent changes in the epidemiology of meningococcal disease in the world, and analyze the development and impact of new conjugate vaccines on the prevention of meningococcal disease. They draw attention to the importance of the administration of a booster dose after 12 months of age to ensure long-term protection. The authors also analyze the different immunization strategies used with these new vaccines, and suggest a more costeffective schedule for its incorporation in the routine immunization schedule of countries such as Brazil.

The benefits of the BCG vaccine in the prevention of tuberculosis are discussed in the article "BCG vaccine: efficacy and indications of vaccination and revaccination," in which the authors conclude that there is no clear evidence that a second dose of the BCG vaccine increases its protective effect.

An up-to-date and critical review of preventive measures against hepatitis $A$ and $B$ is presented in the article "Viral hepatitis prevention by immunization."

In the article "Impact of pneumococcal conjugate vaccine on the prevention of invasive pneumococcal diseases," the authors describe the direct and indirect benefits found in countries that incorporated the 7-valent conjugated pneumococcal vaccine to their immunization schedule, and suggest its inclusion in the PNI.

The article "Universal use of inactivated polio vaccine" brings an overview of the current situation of poliomyelitis in the world and an analysis of the WHO proposals for this period of transition from global eradication to posteradication. They conclude that to have a world $100 \%$ free of poliomyelitis it will be necessary to remove the virus from circulation, which will only be possible if OPV vaccination is discontinued.

The recent finding that healthy infants are affected by the influenza virus similarly to other risk groups was the rationale for the Brazilian Society of Pediatrics to recommend flu vaccines for children 6 to 23 months of age. ${ }^{5}$ In the article "Safety, immunogenicity and efficacy of influenza vaccine in children," the authors report that inactivated vaccines against influenza have low reactogenicity and that they are effective in the prevention of infection by the influenza virus and in the reduction of 
morbidity, although their immunogenicity and efficacy are variable in children.

Special situations, such as immunosuppression, prematurity, pregnancy and exposure to infectious diseases, increase the risk of developing severe disease or experiencing post-vaccination adverse events. ${ }^{6}$ In the article "Vaccination in special situations," the authors review indications, contraindications and efficacy of vaccination in such conditions. They conclude that, after exposure to infectious diseases, individuals with immunodeficiency or immunosuppression whose postvaccination antibody titers were not assessed should not be considered protected. Available prophylactic measures, including passive immunizations, should be adopted, even for patients previously and adequately vaccinated.

In the article "Varicella vaccines and measles, mumps, rubella, and varicella vaccine," the authors present an upto-date review of efficacy studies, adverse effects, and schedules of the vaccine against varicella and the new combination vaccine against those entities. They report that, once universal vaccination against varicella is incorporated, the chances of exposure to a natural stimulus should decrease, and the administration of booster doses will very likely be necessary, probably with the use of the quadrivalent viral vaccine after its licensure.

The impact of the conjugate vaccine against Haemophilus influenzae type b (Hib) in the epidemiology of Hib invasive disease is analyzed in the article "Haemophilus influenzae type b vaccination: long term protection," in which the authors conclude that current epidemiologic conditions in some countries indicate the need to review vaccination schedules and to adopt the use of a booster dose of Hib vaccine after the first year of life.

Finally, a careful review of the current stage of development of vaccines against streptococcus $B$, herpes zoster, HIV, malaria and dengue concludes that a safe and effective vaccine, or vaccines, may be available in the near future.

We hope that our joint effort to carefully prepare the articles in this supplement is rewarded by having this information on the state of the art of vaccines in the world reach as many pediatricians as possible.

\section{References}

1. Plotkin $S L$, Plotkin SA. A short history of vaccination. In: Plotkin $\mathrm{SA}$, Orenstein WA, editors. Vaccines. 4th ed. Philadelphia: Elsevier; 2004. p. 1-16.

2. World Health Organization. Achieving MDGs with immunizations successes. 2004. http://www.who.int/mdg/goals/goal4/ 050511_immunization_ml.pdf. Access: 06/3/2006.

3. Brasil, Ministério da Saúde. Programa nacional de imunizações - 30 anos. Brasília: Ministério da Saúde, Secretaria de Vigilância em Saúde; 2003.

4. Schiller JT, Lowy DR. Human papillomavirus vaccines. In: Plotkin S, Orenstein WA, editors. Vaccines. 4th ed. Philadelphia: Elsevier; 2004. p. 1259-65.

5. Sociedade Brasileira de Pediatria. Calendário vacinal 2005. http://www.sbp.com.br/show_item2.cfm?id_categoria= 24\&id_detalhe=1848\&tipo_detalhe=s. Access: 06/03/2006.

6. American Academy of Pediatrics. Immunization in special clinical circumstances. In: Pickering LK, editor. 2003 red book: report of the Committee on Infectious Diseases. 26th ed. Elk Grove Village: American Academy of Pediatrics; 2003. p. 54-81. 